\title{
THE STRUCTURE OF DENSE CLOUD CORES
}

\author{
Alwyn Wootten \\ National Radio Astronomy Observatory $\left(\mathrm{NRAO}^{1}\right)$ \\ Edgemont Rd., Charlottesville, Va 22903, USA
}

\section{Things are seldom what they seem. Skimmed milk masquerades as cream. Little Buttercup, H. M. S. Pinafore}

Open slit spectra of planetary nebulae, in which images of the object are recorded in the light of several spectral lines on a single plate, have long proven a useful diagnostic of nebular properties and morphology. Fortunately, the reasonably simple structure of most planetaries greatly aids interpretation of the images. The dust-enshrouded mass-losing asymptotic giant branch stars from which planetaries evolve have now also been imaged at millimeter wavelengths. These high-resolution images have demonstrated the role of photochemistry in molding the composition of circumstellar shells. This powerful techinique is less well-developed as a tool for analyzing the structure of localized density concentrations in molecular clouds, the cores in which stars form. Even pre-astral cores, in which stars have not yet formed, may have an extended and intricate geometry which renders mapping tedious and masks their true structure. Their basic pre-astral structure may be complexly contorted by the character and extent of star formation within them. How, then, does our perception of the structure of a core depend upon the line in whose light it is imaged? Which lines optimally determine physical structure? How should chemical differences, perceived by comparisons of images in different lines, be used to determine the physical characteristics of a core?

In this paper I summarize results of surveys of the cores of the $\rho$ Ophiuchi molecular complex in an attempt to provide preliminary answers to these questions. In this nearby ( $160 \mathrm{pc}$ ) cloud, 78 young stars have been catalogued in the central region alone (Wilking, Lada and Young 1989). Loren (1989) has described 89 dense clumps throughout the complex. Within these clumps, Loren, Wootten and Wilking (1989) have described 12 very dense cores, some of which are currently active sites of star formation. A few appear to be undisturbed pre-astral cores whose structure, hopefully, could reveal how they have formed.

As star formation unleashes an important new energy source in a pre-astral core, the core warms. Conventional gas thermometers fail to monitor the early phases of this change. Lower $\mathrm{CO}$

1 The NRAO is operated by Associated Universities, Incorporated, under cooperative agreement with the National Science Foundation 
transitions fail to penetrate a dense core owing to the optical depth of the envelope. Ammonia fails to supply sufficient accuracy for our needs; its abundance also apparently varies dramatically in some denser cores. A potentially much more sensitive temperature monitor can be established through comparison of emission from optically thin deuterium-bearing molecules to other optically thin isotopomers. The $\left[\mathrm{DCO}^{+}\right] /\left[\mathrm{H}^{13} \mathrm{CO}^{+}\right]$ratio $R$ varies especially strongly over the $15-30 \mathrm{~K}$ temperature range which is of most interest (Wootten. Loren and Snell 1982). To establish this ratio as an effective probe, Butner et al. (1988) compared maps of $\mathrm{DCO}^{+}, \mathrm{H}^{13} \mathrm{CO}^{+}$and warm dust (IRAS $12 \mu \mathrm{m}$ map). They found pockets of depressed $R$ values near known stars, even those currently emitting little energy (e.g.E32, at $0.5 \mathrm{~L}_{\odot}$ ). Using this tool, they were able to pinpoint the location of the pre-astral core B2 (Figure 1). This tool has now been applied to other cores (Loren, Wootten and Wilking 1989) which have now been established as pre- or post-astral. Unfortunately, excitation effects can bias the interpretation of individual maps and several-transition images may be crucial to proper understanding of core structure.

In L1689N, a cold pre-astral core lies adjacent to the warm core harboring the young star IRAS16293-2422 (Wootten and Loren 1986). $\mathrm{DCO}^{+}$maps (Wootten, Butner, Loren, Kaifu, Yamashita and Hayashi, in preparation) show similar emission at both locations, confounding expectations. $R$, measured from the $\mathrm{J}=1-0$ lines, varies insignificantly, increasing from 1.7 at the IR source to 2 at the cold core. In contrast, the warm core vanishes in images in the $J=3-2$ $\mathrm{DCO}^{+}$line (Wootten and Loren 1986). An image in the $\mathrm{J}=3-2 \mathrm{HCO}^{+}$line barely shows the cold pre-astral core, so dominated is it by powerful emission from the warm core. Here, clearly, excitation plays a major role in the determination of the structures of these cores. However, when the effects of excitation are properly accounted, it is clear that the deuterium enhancement in $\mathrm{HCO}^{+}$provides an excellent identifler of cold dense pre-astral cores where stars have not yet formed.

Ammonia maps also reveal interesting aspects of chemical differentiation within L1689N. Single-antenna maps of the $(1,1)$ line (Wootten and Loren 1986) and the $(2,2)$ line (unpublished data) show a cloud completely dominated by emission from the cold core. Measures of the cloud mass, using a standard ammonia analysis (Ho and Townes 1983) fix the cold core mass at $6 \mathrm{M}_{\odot}$, while the warm star-forming core contains less than $0.1 \mathrm{M}_{\odot}$ ! Not surprisingly, perhaps, high resolution maps (Mundy, Wootten and Wilking, 1989) of other mass tracers require much more material in the post-astral core. Using various estimators, masses range from $>0.22 \mathrm{M}_{\odot}$ (from $\mathrm{C}^{18} \mathrm{O}$ flux measurements) to $1.6 \mathrm{M}_{\odot}$ (suggested by the dynamics of disk rotation which includes a central object) to $0.9-6 \mathrm{M}_{\odot}$ (from dust emission measurements). High resolution maps of the $(1,1)$ lines of $\mathrm{NH}_{3}$ reveal a hole in emission near the central source, where $\mathrm{C}^{18} \mathrm{O}$ and dust emission peaks. Nowhere does the temperature derived from high resolution $(2,2)$ ammonia observations 


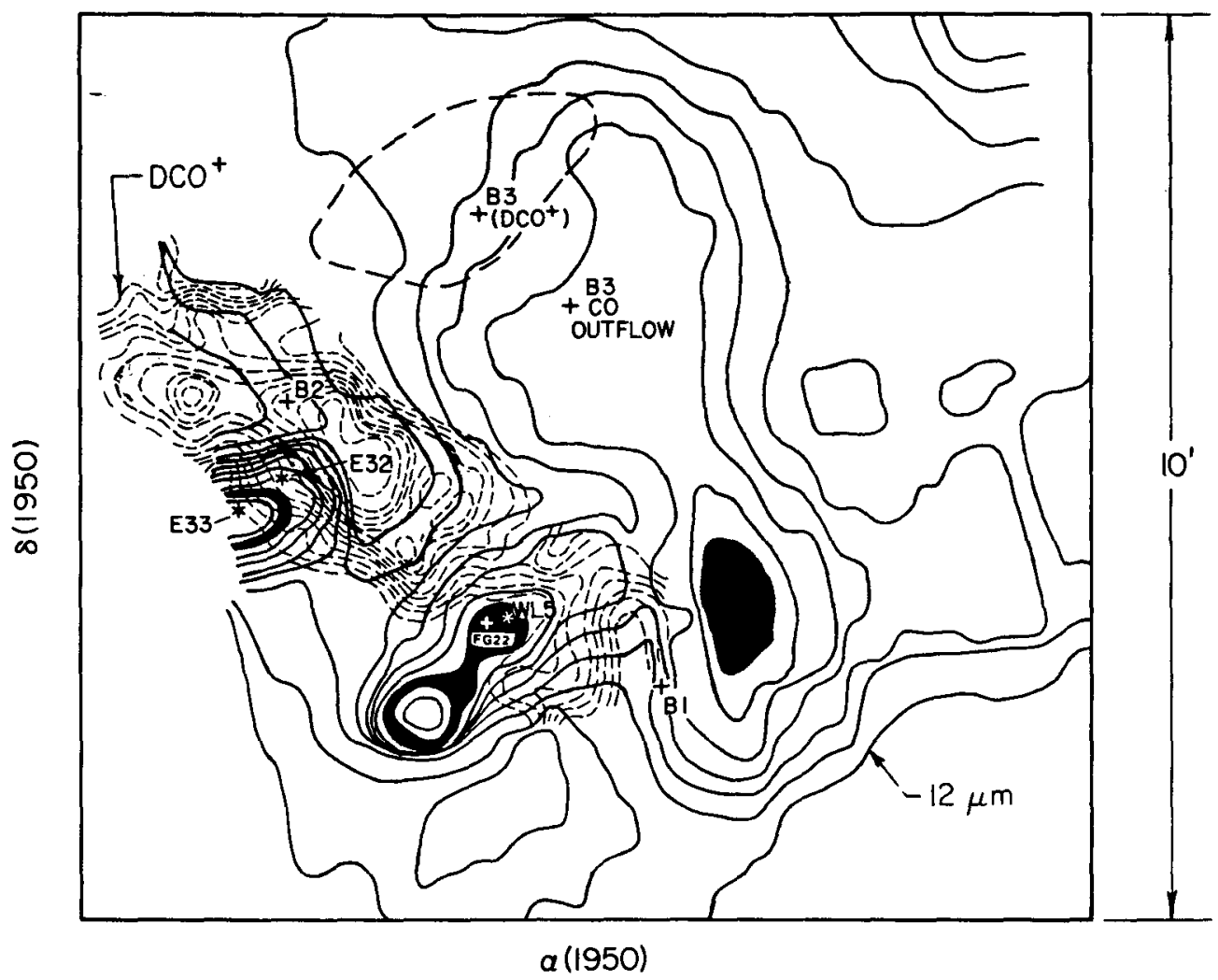

Fig. 1. Map of the region around the pre-astral core $\rho$ Oph B2 (Butner et al. 1988). Solid contours, taken from IRAS $12 \mu \mathrm{m}$ maps, trace warm dust. Highlighted contours show local peaks near point sources Elias 32 and 33, WL5, and extended source YLW10A (right of B1). At E32 and E33, $\mathrm{H}^{13} \mathrm{CO}^{+} \mathrm{J}=1-0$ emission peaks, demonstrating a gas column density enhancement occurs there, as well as a dust temperature peak. Light dashed contours trace $\mathrm{DCO}^{+} \mathrm{J}=1-0$ intensity, obtained with 15" resolution. Note that these contours avoid E32 and E33. $R$ then locates the unheated material and defines core B2. Near the most luminous source in the region, WL5 and its thermal radio source FG22, the column density of all measured molecules declines, illustrating the evacuation of molecules from its vicinity. Dense core B1, and modest density core B3, defined by lower resolution $\mathrm{DCO}^{+}$observations, are marked, as is a region of low-velocity $\mathrm{CO}$ flow of uncertain origin near $\mathrm{B3}$. 
exceed $15 \mathrm{~K}$, reinforcing our conclusion that ammonia emission is lacking from the central disk. Lastly, ammonia only weakly reveals the strong velocity field of the disk, a striking characteristic of the $\mathrm{C}^{18} \mathrm{O}$ maps. The most plausible reason for the disappearance of ammonia emission is that the ammonia molecules have accreted onto grains and core warming has not sufficiently progressed to release them back to the interstellar gas. One can only conclude that ammonia offers an exceptionally poor probe of the densest regions of this cloud core.

Next, consider the emission from sulfur-bearing molecules in these cores. Sulfur chemistry is initiated by endothermic reactions. Hence it could offer a foil to the cold-sensitive deuterium enrichment probe discussed earlier. A reasonable working hypothesis holds that maps of sulfur compounds will pinpoint regions of active star-forming activity. Wootten and Loren (1988, and in preparation) measured several low-lying transitions of $\mathrm{SO}_{2}$ and $\mathrm{SO}$ in the Oph $\mathrm{A}, \mathrm{Oph} \mathrm{B1}$ and B2, and IR16293 cores. $\mathrm{C}^{34} \mathrm{~S}$ emission was measured as a control against which to contrast possible changes in relative abundance. Within the B1 and IR16293 cores, and at the peak of $1.3 \mathrm{~mm}$ dust emission in $\mathrm{Oph} \mathrm{A}$, the $\left[\mathrm{SO}_{2}\right] /\left[\mathrm{C}^{34} \mathrm{~S}\right]$ ratio was stable, showing neither enhancement nor depression. However, near the young star GSS30, lying northwest of the Oph A core, strong emission from $\mathrm{SO}_{2}$ and $\mathrm{SO}$ was found, in a region where emission from no other molecules was known to peak. The line brightness temperature in the unresolved region lies near $8 \mathrm{~K}$ in the $3_{13}-2_{02}$ line, similar to the excitation temperature measured between this and the $3_{22}-2_{11}$ line, indicating the line could be optically thick. These observations require at least an order of magnitude enhancement of the $\mathrm{SO}_{2}$ abundance at GSS30 over other positions in the cloud. It is difficult to attribute this to a shock, although high-velocity wings are known in $\mathrm{CO}$ (Loren et al. 1980 ) and $\mathrm{HCO}^{+}$(Loren and Wootten 1982) at a position to the southwest. The strong $\mathrm{SO}_{2}$ line shows a profile substantially narrower $\left(0.8 \mathrm{~km} \mathrm{~s}^{-1}\right)$ than the $\mathrm{C}^{34} \mathrm{~S}$ line $\left(1.6 \mathrm{~km} \mathrm{~s}^{-1}\right)$ at GSS30! Although our expectation that $\mathrm{SO}_{2}$ would pinpoint active star-forming regions appears justified, a detailed explanation for the character of the emission remains perplexing.

Lastly, we consider an oft-used density probe, the $2 \mathrm{~cm}$ line of formaldehyde. Usually seen in absorption against the cosmic background radiation, in fairly dense regions $\left(\mathrm{n}\left(\mathrm{H}_{2}\right) \sim 10^{6} \mathrm{~cm}^{-3}\right)$ the line appears in emission. Only a few such regions are known-in OMC1 and OMC2, DR21(OH) and in the Oph B core. A recent survey (Wootten, Butner, Mangum and Loren in preparation) has revealed two more emission regions in the $\rho \mathrm{Oph}$ complex. One is associated with a dense clump of gas which contains an embedded radio continuum source, L1689S. This clump also emits strongly in the $1.3 \mathrm{~mm}$ formaldehyde lines. An excitation analysis confirms that this clump is quite dense. As in the other regions where it has seen, here the $2 \mathrm{~cm}$ line has proven an effective locator of a dense clump, probably active in star formation. The second region, however, is more enigmatic. Lying considerably east of both the cold and warm cores in L1689N, there is only 
weak emission in the millimeter formaldehyde lines at its position. An excitation analysis fails to account for its formaldehyde spectrum. Ammonia emission is also quite weak, again suggesting high densities are absent. It would seem quite difficult to argue on any line of evidence except that of the $2 \mathrm{~cm}$ emission that a dense clump was located here. As with the other probes of core structure, the $2 \mathrm{~cm}$ line also appears to have vexing exceptions to its general usefulness as a density probe.

We conclude that indeed the perception of the structure of dense cores depends critically upon the transition in whose light it is imaged. Although a number of probes seem useful for discriminating cores harboring active star formation from those which are quiet, in each situation a complexity enters the interpretation. Furthermore, each core illustrates a different stage of star formation. Only with suitably detailed maps of well-chosen molecules will we be able to piece together the whole time sequence to describe the changes in cloud structure which herald core formation and presage the creation of a new star.

\section{REFERENCES}

Butner, H. M., Wootten, A., Loren, R. B., Kaifu, N., Suzuki, H., Yamashita, T. and Hayashi, S. 1988 in Molecular Clouds in the Milky Way and External Galaxies, ed. R. Dickman, R. Snell and J. Young, Springer-Verlag: Berlin, p. 32.

Ho, P. and Townes, C. 1983 Ann. Rev. Astr. Ap., 21, p. 239.

Loren, R. 1989 Ap. J., 338, 902.

Loren, R. and Wootten, A. 1982, ESA SP-192 Galactic and Extragalactic Infrared Spectroscopy, p. 93 .

Loren, R. and Wootten, A. 1986 Ap. J., 306, 142.

Loren, R., Wootten, A. and Wilking, B. 1989, submitted.

Loren, R., Wootten, A., Sandqvist, A., and Bernes, C. 1980, Ap. J. (Letters), 240, L65.

Mundy, L., Wootten, A. and Wilking, B. 1989, in preparation.

Wilking, B., Lada, C. and Young, E. 1989 Ap. J., 340,, 823.

Wootten, A. and Loren, R. 1987 Ap. J., 317, 220.

Wootten, A. and Loren, R. 1989 in The Physics and Chemistry of Interstellar Molecular Clouds, ed. T. Armstrong and G. Winnewisser, in press.

Wootten, A., Loren, R. B., and Snell, R. L. 1982, Ap. J., 255, 160. 